\title{
Evaluación de la actividad antimicrobiana de bacterias lácticas aisladas de productos lácteos comerciales frente a Escherichia coli
}

\section{Evaluation of the antimicrobial activity of lactic acid bacteria isolated from artisanal and commercial dairy products against Escherichia coli}

Bryan Leal Rojas ${ }^{1}$ y Claudia Milena Amorocho Cruz ${ }^{2}$

\begin{abstract}
Resumen
Las Bacterias Acido Lácticas (BAL) se destacan por su capacidad de producir sustancias de actividad antimicrobiana como ácidos orgánicos, peróxido de hidrógeno y bacteriocinas, es por esta razón que se realizó una búsqueda, aislamiento e identificación de cepas BAL provenientes de yogurt y queso hilado de producción artesanal y en producción comercial en la región del Huila en Colombia, una vez aisladas se evaluó la actividad antimicrobiana frente a cepas de patógenos de Escherichia coli (aislada del restaurante de la Universidad Surcolombiana y de una venta ambulante de ají en tres diferentes métodos: presencia de células (método de discos), ausencia de células (método de pocillos) con pH ácido y neutro. Los resultados obtenidos en la actividad antimicrobiana por parte de las BAL, abre la posibilidad de ofrecer a la industria de lácteos local semi-industrial, artesanal y a los consumidores, información que les ayude a la producción de alimentos (Yogurt y Quesillo) con atributos de calidad, y potencial probiótico con actividad antimicrobiana, posicionándolos como un alimento funcional. Los resultados de la caracterización físico química de los productos lácteos (Yogurt y Quesillo), elaborados y distribuidos de manera artesanal y semi-industrial en el departamento del Huila, son de gran relevancia no solo para garantizar su calidad y aceptabilidad en el mercado para los consumidores; sino también como una herramienta de identidad útil para clasificar los diversos tipos de quesillos y yogurts.
\end{abstract}

Palabras clave: probióticos; patógenos; inhibición.

\footnotetext{
${ }^{1}$ Ingeniero Agrícola. Universidad Surcolombiana, Grupo de investigación USCO agroindustria. mecanicadesuelos.20141@gmail.com

${ }^{2} \mathrm{PhD}$ profesor. Universidad Surcolombiana. Grupo de investigación USCO agroindustria. Claudiamilena.amorocho@usco.edu.co
} 


\begin{abstract}
Lactic acid bacteria (LAB) stand out for their ability to produce substances with antimicrobial activity such as organic acids, hydrogen peroxide and bacteriocins, therefore, the isolation and identification of LAB strains was conducted from yoghurt and cheese of artisanal or commercial elaboration from the Huila Department of Colombia. Once isolated antimicrobial activity was evaluated against strains of pathogenic Escherichia coli (isolated from restaurant Surcolombiana University and a peddling aji. using different methods: presence of cells (disk method), absence of cells (well method) with neutral and acid $\mathrm{pH}$. The results obtained in the antimicrobial activity by the BAL, opens the possibility of offering to the local semi-industrial, artisanal dairy industry and to consumers, information that will help them to produce food (Yogurt and Quesillo) with attributes of quality, and probiotic potential with antimicrobial activity, positioning them as a functional food. The results of the physical and chemical characterization of dairy products (Yogurt and Quesillo), elaborated and distributed in an artisanal and semi-industrial way in the department of Huila, are of great relevance not only to guarantee their quality and acceptability in the market for the consumers; but also as a useful identity tool to classify the different types of quesillos and yogurts.
\end{abstract}

Keywords: probiotics; pathogens; inhibition.

\section{Introducción}

Las Bacterias Acido Lácticas (BAL), han estado presentes en la alimentación desde hace siglos; se encuentran en productos fermentados como la leche y derivados, productos cárnicos y vegetales; las cuales proporcionan sabor y textura e incrementan el valor nutricional de los alimentos (Mateos, 2002). Estos microorganismos cuando fermentan carbohidratos producen una mezcla de sustancias con acción antimicrobiana como: ácido láctico, ácido acético, ácido butírico, peróxido de hidrógeno, diacetilo y péptidos de bajo peso molecular llamados bacteriocinas, que generan cambios en la microbiota intestinal como la inhibición de patógenos; lo cual repercute positivamente en el estado de salud del consumidor (Casas et al., 2000). Por ello las BAL son alternativa en el tratamiento de infecciones bacterianas tópicas o sistémicas por microorganismos resistentes a la mayor parte de los antibióticos conocidos, así tienen la capacidad de contribuir a la disminución de resistencias frente a los mismos (Jamuna, 2004).

Un alimento funcional puede ser un alimeno natural, al que se le ha añadido, eliminado o modificado un componente por medios biotecnológicos, un alimento en el que se ha modificado la biodisponibilidad de uno o más de sus componentes o una combinación de cualquiera de estas posibilidades (Diplock et al., 1999; Isolauri et al., 2000). El ejemplo más claro de un alimento funcional es la leche humana, que contiene gran número de elementos bioactivos (enzimas, factores de crecimiento, aminoácidos libres, inmunoglobulinas, oligosacáridos, etc.) cuyo efecto va mucho más allá del puramente nutricional. Existen muchos componentes de alimentos de los que se ha demostrado su efecto beneficioso sobre determinadas funciones del organismo. La adición de estos componentes a un alimento, lo transforma en un alimento funcional, por ejemplo, el yogurt con bacterias probióticas, el ácido fólico en los cereales o los ácidos grasos en las margarinas y productos lácteos. De todos ellos cabe destacar el grupo de los probióticos, prebióticos y simbióticos, (Metchnikoff et al., 1908).

Entre los géneros de las BAL más utilizadas para el consumo humano se encuentran los siguientes: Lactococcus, Streptococcus, Bifidobacterium, Enterococcus, Lactobacillus, entre otros (Farnworth, 2001). El género Lactobacillus está conformado por microorganismos reconocidos por su habilidad fermentativa e iniciadores en procesos industriales de productos fermentados; su presencia en el tubo digestivo es considerada benéfica, por tener un papel protector o terapéutico (Jiang, 1996). Según (Mustapha et al., 1996) se ha visto un aumento en la adición de BAL en productos comerciales reconocidos, con el fin de dar un valor agregado al producto y a la vez mejorar los procesos y aumentar la estabilidad y tiempo de conservación de los productos.

Es por esta razón que se propone realizar una búsqueda, aislamiento e identificación de cepas BAL presentes en productos lácteos artesanales y comerciales como yogurt y quesillos producidos en el sur de Colombia, adicionalmente realizar una evaluación in vitro para identificar las cepas que puedan presentar actividad antimicrobiana frente a cepas de Escherichia coli. 


\section{Materiales y Métodos}

\section{Recolección de muestras}

Se seleccionaron muestras de yogurt y queso hilado elaborados y empacados artesanal y comercialmente en la región de Huila en Colombia; Yogurt y Quesillo. Artesanalmente las muestras correspondieron a yogurt elaborado en el Caguán (Ya), y quesillos de $250 \mathrm{~g}$ fabricados en el municipio de Hobo (Qa). Industrialmente las muestras correspondieron a yogurt fabricado por una empresa regional (Yc), y quesillos de $250 \mathrm{~g}$ fabricados por una reconocida empresa Huilense (Qc). Todas las muestras se obtuvieron por triplicado y fueron transportadas el mismo día de su elaboración, en termos refrigerados hasta el laboratorio de microbiología de alimentos de la Facultad de Ingeniería de la Universidad Surcolombiana, siendo allí donde se realizaron las pruebas físicas, químicas y microbiológicas correspondientes.

\section{Caracterización Fisicoquímica}

Se realizaron pruebas de caracterización fisicoquímica en todas las muestras de Ya, Yc, Qa y Qc, el pH en quesos se realizó de acuerdo a (Gonzales, 2010) con potenciómetro (WTW -330) previamente calibrado de acuerdo a la norma (AOAC-981.12-1997), el pH del Yogurt se realizó directamente a una muestra de $50 \mathrm{ml}$ la acidez titulable en queso se realizó de acuerdo a (Meyer et al., 1982), citado por Gonzales (2010) para la determinación de este parámetro en Yogurt se homogenizó $18 \mathrm{~g}$ de muestra con $36 \mathrm{~g}$ de agua destilada, se añadió cinco gotas de Fenolftaleína y se tituló con hidróxido de sodio 0.1 N. Se determinó el contenido de humedad de las muestras de quesillo mediante el método gravimétrico indirecto (García \& Fernández, 2012), establecido por la AOAC 926.08 (1997). Para Yogurt se midió solidos solubles, con ayuda del refractómetro (ATAGO PR-201a).

\section{Aislamiento BAL}

\section{Quesillo hilado (Qa y Qc)}

Se tomó una muestra de quesillo de $10 \mathrm{~g}$ y se homogenizó en $90 \mathrm{ml}$ de solución estéril de caldo MRS (bacterias lácticas) (Dibico), para luego efectuarse diluciones seriadas. Se sembró en masa alícuotas de $1 \mathrm{ml}$ en agar MRS (MERCK) por triplicado, incubados durante 48 h a $37^{\circ} \mathrm{C}$.
Transcurrido el tiempo se seleccionaron las colonias más representativas según color, tamaño y textura para realizar un aislamiento puro.

\section{Yogurt (Ya y Yc)}

Se tomó una muestra de $1 \mathrm{ml}$ y se homogenizó en 9 $\mathrm{ml}$ de caldo MRS (bacterias lácticas) (Dibico), para luego efectuarse diluciones seriadas. Se sembró en masa alícuotas de $1 \mathrm{ml}$ en agar MRS (MERCK) por triplicado, luego fueron incubados durante $48 \mathrm{~h}$ a $37^{\circ} \mathrm{C}$ en condiciones anaeróbicas. Transcurrido el tiempo se seleccionaron las colonias más representativas según color, tamaño y textura para realizar un aislamiento puro.

\section{Descripción morfológica de BAL}

Para la identificación y descripción morfológica de las bacterias aisladas de los dos productos seleccionados se realizó Tinción Gram, y posterior observación en microscopio (OLYMPUS CH-.2).

\section{Evaluación antimicrobiana de las BAL frente a Escherichia coli}

Esta evaluación se realizó a 12 cepas BAL aisladas de yogurt y quesillo fabricados y distribuidos de forma artesanal e industrial (Ya, Yc y Qa, Qc). Se estudió el impacto de la presencia y ausencia de células BAL frente a cepas de Escherichia coli. En la tabla 1 se muestra el origen del que fueron aisladas las cepas de éste patógeno según (Pérez et al.,2015).

El patógeno Escherichia coli fue aislado de una venta ambulante de arepas y del restaurante de la Universidad Surcolombiana.

Para la activación de estas cepas de patógeno se sembró en estría cada uno de ellos en medios selectivos Agar XLD (Agar-Xilosa-LisinaDesoxicolato) (DIBICO), Agar de ENDO (DIBICO) y Agar Salmonella Shigella (DIBICO); incubados durante 24 horas a $37^{\circ} \mathrm{C}$.

\section{Método de Discos, Presencia de células BAL.}

Se realizó la evaluación antimicrobiana en presencia de células BAL, para ello se empleó la técnica de Discos según (Yuan, 2006) con algunas modificaciones hechas por Amorocho (2011). Las cepas de cada patógeno activados se sembraron en agar Plate Count (PC) con ayuda de un asa estéril y 
tomando solo una pequeña porción de ésta. De los cultivos puros de BAL a 24 horas en agar MRS obtenidos del aislamiento, se obtuvieron discos de 8 mm de diámetro cortándolos directamente con un sacabocados estéril. Los discos se depositaron sobre la placa de agar PC donde previamente se había extendido el respectivo patógeno. Las placas se incubaron a $37^{\circ} \mathrm{C}$ durante 48 horas. Este método se evaluó por triplicado.

\section{Método del sobrenadante en pocillos, Ausencia de células BAL.}

Este método se utilizó para evaluar la inhibición de los patógenos debido a los productos generados por las BAL y en ausencia de las células lácticas (Yuan, 2006) con algunas modificaciones hechas por Amorocho (2011). Con el propósito de eliminar las células, el sobrenadante obtenido se esterilizó mediante el uso de filtros con membrana de nitrocelulosa y $0.22 \mu \mathrm{m}$ de tamaño de poro (Milipore, Bedford, MA). Los ensayos con sobrenadante se realizaron a $\mathrm{pH}$ a 4.5 y a $\mathrm{pH} 7$ ajustando con $\mathrm{NaOH}$ $1 \mathrm{~N}$ para cada patógeno, por triplicado.

Cada cepa de BAL, fue evaluada por su poder antimicrobiano frente a las dos cepas de patógeno seleccionado, en tres diferentes métodos: Presencia de células (1), ausencia de células $\mathrm{pH}$ acido (2), ausencia de células $\mathrm{pH}$ neutro (3).

\section{Análisis Estadístico}

El análisis estadístico se hizo con el empleo del programa informático StatGraphics plus 5.1. Se realizó un análisis de varianza simple (ANOVA) para los datos de inhibición en cada uno de los tratamientos y entre tratamientos, el cual efectuó varios tests y gráficos para comparar los valores medios entre los diámetros de inhibición para los tres tratamientos. En caso de presentarse valores atípicos se elige el test no paramétrico de Kruskal-Wallis que compara las medianas en lugar de las medias. Las diferencias entre medias o medianas fueron consideradas significativas cuando $\mathrm{p}<0.05$.

\section{Resultados y Discusión}

\section{Análisis Fisicoquímicos del Quesillo}

En la tabla 2 se muestra las características iniciales de las muestras de quesillo.

Tabla 2. Caracterización de las muestras de Quesillo hilado

\begin{tabular}{ccc}
\hline PARÁMETROS & $(\mathrm{Qa})$ & $(\mathrm{Qc})$ \\
\hline $\mathrm{pH}$ & 5.798 & 6.206 \\
$\begin{array}{c}\text { Acidez Titulable } \\
\text { (\% A. láctico) }\end{array}$ & 0.255 & 0.135 \\
Humedad (\%) & 49.242 & 45.544 \\
\hline
\end{tabular}

Se puede observar un $\mathrm{pH}$ ligeramente ácido para ambas muestras, los valores de humedad obtenidos resultaron más elevados (43-50\%) que los publicados para queso fresco (Ramírez \& Vélez, 2012); en general, la caracterización inicial encontrada para estas muestras de quesillo recién fabricado presenta variación con respecto a los presentados por (Cortes et al.,2016) para quesillo colombiano y por (Oliszewski et al.,2007) para quesillo Argentino.

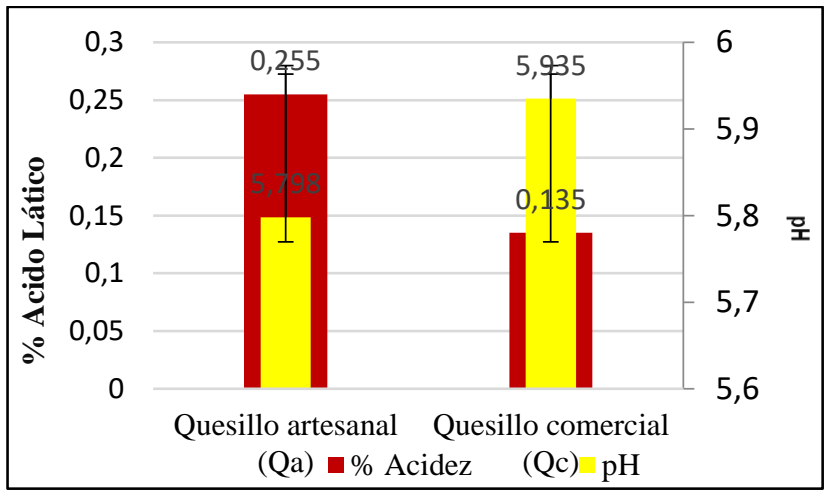

Figura 1. Valores promedios de acidez y $\mathrm{pH}$ en quesillos (QA, Qc)

Se observa en la Figura 1 que a menor $\mathrm{pH}$ (5.798) mayor acidez $(0.255 \%$ de A.L) el cual fue característico de la muestra (Qa), y que a mayor valor de $\mathrm{pH}$ (6.206), el valor de acidez disminuyó (0.135\% de A.L), en (Qc), guardando una relación inversa entre las dos variables. La diferencia en valores de acidez y $\mathrm{pH}$ de cada producto dependen de la temperatura ambiente y del tiempo de acidificación que se da más rápido en la ciudad de Neiva $\left(38^{\circ} \mathrm{C}\right.$ 
promedio), debido a que las bacterias descomponen más rápido el sustrato a temperaturas mayores a 35 ${ }^{\circ}$ C. Según (Castillo, 2001), los quesillos presentan valores de $\mathrm{pH} 5.3$ y acidez de 0.91 más o menos 0.07 ; lo cuales son cercanos a los rangos de $\mathrm{pH}$ presentados en este trabajo (5.798 para Qa y 5.935 para Qc) mientras que los valores de $\%$ acidez de los quesillos evaluados están por debajo (0.255-0.135 \% A.L).

En una investigación realizada por (Maldonado \& Llanca 2008) en quesos de mano venezolanos se encontró que el $\mathrm{pH}$ fue de 4.51 y la acidez de 0.76 , los valores de $\mathrm{pH}$ y acidez están fuera de los rangos obtenidos en esta investigación. (Bennik, 1999) publicó para $\mathrm{pH}$ rangos de 4.90 a 5.85 , los cuales están entre los valores de $\mathrm{pH}$ obtenidos en este estudio para Qa. Por otra parte, (Jay, 2000) obtuvo en acidez valores de 0.47 a $0.53 \%$ estando muy por encima de los valores reportados por los quesillos (Qa y Qc) del departamento del Huila.

En general, la humedad de los quesillos elaborados (Qa, Qc), están en un rango elevado entre 45 y $50 \%$ como se observa en la Tabla 2 , lo que permite clasificarlos como quesillos frescos que según su consistencia se clasifican en quesos blandos, dado que tienen una humedad mayor del $45 \%$ (Ramírez et al., 2010).

\section{Caracterización del Yogurt}

En la tabla 3 se muestra que los valores de $\mathrm{pH}$ y acidez titulable (\% ácido láctico) de (Ya, Yc) fueron 4,$625 ; 3,905$ y 0,$728 ; 0,860$; respectivamente; valores aceptables para comercialización; dado que los valores de acidez titulable están dentro del rango establecido por la norma NTP: 202.192. 2014 (INDECOPI-PERÚ, 2014), de 0,6 a 1,5\%, para la acidez del yogur de leche de vaca. Asimismo, otras normas como CODEX STAN 243-2003 (FAO, 2003) y NOM-181-SCFI-2010 (DGN MEXICO, 2010) para yogur de leche de vaca, señalan una acidez mínima de $0,6 \%$ y de $0,5 \%$, respectivamente; y al ser el $\mathrm{pH}$ una medida influenciada por la acidez se puede afirmar que los valores de $\mathrm{pH}$ medidos son adecuados.
Tabla 3. Caracterización de las muestras de Yogurt

\begin{tabular}{ccc}
\hline PARÁMETROS & (Ya) & (Yc) \\
\hline $\mathrm{pH}$ & 4.600 & 3.905 \\
Acidez Titulable (\% A. láctico) & 0.728 & 0.860 \\
solidos solubles & 17.600 & 14.400
\end{tabular}

(Schimidt et al., 2012) señala que el rango de $\mathrm{pH}$ entre 4,0 y 4,6 se considera más cercano al ideal para yogur de leche de vaca, ya que el producto en este intervalo de $\mathrm{pH}$ no presenta un sabor demasiado amargo o agrio, en los resultados de esta investigación sólo las muestras (Ya) están dentro del rango, debido a que en la elaboración de este producto su fermentación se detuvo cuando el $\mathrm{pH}$ se encontraba en estos valores y con un \% de acidez titulable de 0.70 a 0.80 .

\section{Aislamiento e identificación de Bacterias Acido lácticas (BAL)}

En la Figura 2 se observa las medias del crecimiento de BAL medido en Logaritmo en base 10 unidades formadoras de colonias por gramo ( $\mathrm{Log}$ $\mathrm{UFC} / \mathrm{g}$ ) en función del producto y tipo de fabricación, se muestra que a un nivel de confianza del $95 \%$ se obtuvo diferencias estadísticamente significativas entre el crecimiento de UFC entre muestras de quesillo (Qa, Qc) y entre muestras de yogurt (Ya, Yc)

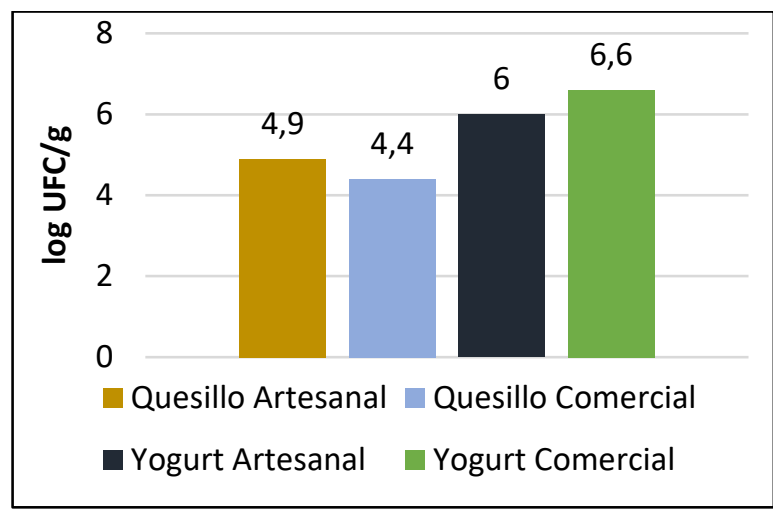

Figura 2. Crecimiento de BAL medido en Log UFC/g de Qa, Qc, Ya y Yc

Se obtuvieron 3 cepas de BAL aisladas por cada producto, obteniendo en total 12 cepas. Su caracterización morfológica comprueba que éstas 
corresponden a bacterias que observadas al microscopio dan coloración GRAM (+) correspondiente aBAL, las cuales se identificaron como: Q.A-3T2, Q.A-5T3, Q.A-7T3, Q.C-1T1, Q.C2T2, Q.C-1T2, Y.A-3T1, Y.A- 5T2, Y.A-3T2, Y.C5T2, Y.C-5T1 y Y.C-4T2, agrupadas en cadenas de cocos, cocos diplococos y bacilos.

\section{Evaluación de la Actividad Antimicrobiana}

Se observa en la Tabla 4 y de acuerdo al análisis estadístico, el test de Kruskal-Wallis aplicado entre los métodos 1, 2 y 3 mostraron diferencias estadísticamente significativas $(\mathrm{p}<0.05)$ entre los métodos utilizados, resaltando que por el método 1 de evaluación antimicrobiana se obtuvieron mayores diámetros de inhibición frente a las 4 cepas de patógenos en estudio, y el método 3 presentó los menores diámetros de inhibición, lo cual hizo diferir los resultados frente a los otros dos métodos.

Tabla 4. Comparación de los diámetros de inhibición frente a los tres métodos de evaluación

\begin{tabular}{cccc}
\hline \multicolumn{4}{c}{ Diámetros de Inhibición } \\
\hline Método & $\overline{\boldsymbol{X}} \pm \mathbf{S}$ & $\mathbf{M e} \pm \mathbf{S}$ & $\mathbf{P}$ \\
& & & valor \\
1 & $11,701 \pm 2,335^{\mathrm{a}}$ & $12,0 \pm 2,335^{\mathrm{a}}$ & \\
2 & $9,993 \pm 2,846^{\mathrm{b}}$ & $10,0 \pm 2,846^{\mathrm{b}}$ & 0,0 \\
3 & $7,618 \pm 1,608^{\mathrm{c}}$ & $7,0 \pm 1,608^{\mathrm{c}}$ & \\
\hline
\end{tabular}

Letras minúsculas diferentes indican diferencias estadísticamente significativas entre tratamientos con $\mathrm{P}=0,0$

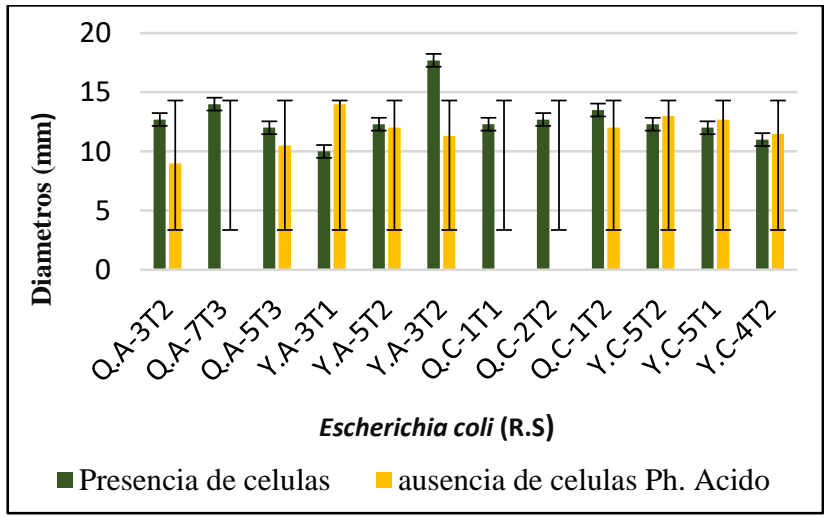

Figura 3. Actividad antimicrobiana de BAL frente a Escherichia coli (R.S) por los 3 métodos

En la Figura 4 se observa el comportamiento de los diámetros de inhibición de cada una de las cepas de BAL frente al patógeno $E$. coli (V.A.A), corroborando que frente a este patógeno el $100 \%$ de las BAL evaluadas con el método 1, presentaron inhibición aunque con diámetros de menor tamaño (10.3-15.3 mm), el 75\% indicaron inhibición con el método 2 con tamaños de 10.5-14 mm en diámetro, $\mathrm{y}$ $16,7 \%$ de BAL tuvieron poder antagónico por el método 3, resaltando que frente a éste patógeno las células de las bacteriocinas si actuaron como agente inhibidor.

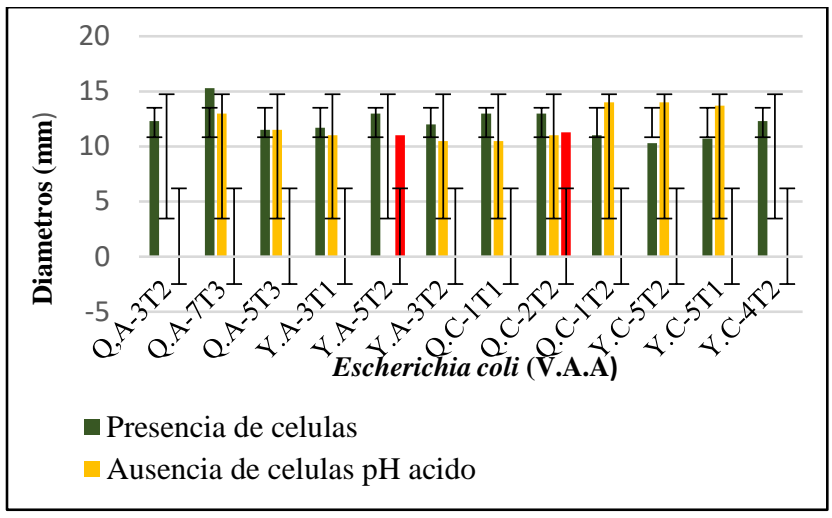

Figura $\mathbf{N}^{\circ}$ 4. Actividad antimicrobiana de BAL frente a Escherichia coli (V.A.A) por los 3 métodos

En la tabla 5 se observa que, de acuerdo al análisis estadístico, el test de Kruskal-Wallis aplicado entre las cepas BAL y su efecto en los diámetros de inhibición, mostró no diferencia estadísticamente significativa $(p<0.05)$ entre las BAL, esto resalta el valor agregado de los productos lácteos elaborados y empacados artesanalmente, pues al igual que los comerciales o industriales presentan características probióticas, con actividad antimicrobiana frente a los patógenos evaluados, incrementando su nivel de competitividad y valor benéfico en la salud del consumidor.

Tabla 5. Comparación del efecto de BAL en los diámetros de inhibición

\begin{tabular}{cccc}
\cline { 2 - 3 } BAL & \multicolumn{2}{c}{ Diámetros de Inhibición } & P valor \\
\hline Q.A-3T2 & $9,111 \pm 2,758^{\mathrm{a}}$ & $7,0 \pm 2,758^{\mathrm{a}}$ & \\
Q.A-5T3 & $9,333 \pm 3,535^{\mathrm{a}}$ & $7,0 \pm 3,535^{\mathrm{a}}$ & \\
Q.A-7T3 & $9,444 \pm 2,455^{\mathrm{a}}$ & $10,0 \pm 2,455^{\mathrm{a}}$ & \\
Q.C-1T1 & $8,844 \pm 2,373^{\mathrm{a}}$ & $7,0 \pm 2,373^{\mathrm{a}}$ & \\
Q.C-2T2 & $9,488 \pm 2,966^{\mathrm{a}}$ & $7,0 \pm 2,966^{\mathrm{a}}$ & \\
Q.C-1T2 & $9,688 \pm 3,182^{\mathrm{a}}$ & $7,0 \pm 3,182^{\mathrm{a}}$ & 0,071 \\
Y.A-3T1 & $9,444 \pm 2,904^{\mathrm{a}}$ & $7,0 \pm 2,904^{\mathrm{a}}$ & \\
Y.A-5T2 & $10,08 \pm 2,538^{\mathrm{a}}$ & $10,0 \pm 2,538^{\mathrm{a}}$ & \\
Y.A-3T2 & $10,80 \pm 3,279^{\mathrm{a}}$ & $11,0 \pm 3,279^{\mathrm{a}}$ & \\
Y.C-5T2 & $10,64 \pm 2,805^{\mathrm{a}}$ & $11,0 \pm 2,805^{\mathrm{a}}$ & \\
Y.C-5T1 & $10,20 \pm 2,865^{\mathrm{a}}$ & $11,0 \pm 2,865^{\mathrm{a}}$ & \\
Y.C-4T2 & $9,02 \pm 2,241^{\mathrm{a}}$ & $7,0 \pm 2,241^{\mathrm{a}}$ & \\
\hline
\end{tabular}


La cepa de Yogurt Artesanal Y.A-3T2, destacó dentro de la colección de las BAL porque tiene actividad antimicrobiana frente a las dos cepas de Escherichia coli con los métodos 1 y 2 . Además esta cepa es la que presenta el diámetro mayor $(17.7 \mathrm{~mm})$ el cual es obtenido frente a la cepa patógena Escherichia coli (R.S) en presencia de células (método de Discos). Por tanto, se considera que su actividad antagónica se debe a la presencia de las células, componentes celulares, a los ácidos orgánicos producidos como el ácido láctico y las cepas que mantuvieron la actividad antimicrobiana a $\mathrm{pH}$ neutro se debe a la producción de otras sustancias activas como las bacteriocinas, como ya concluyeron (Midolo et al., 1995) y (Jacobsen et al., 1999) en estudios similares.

Todas las cepas de las BAL de los dos productos lácteos (Qa, Qc, Ya y Yc) presentaron actividad inhibitoria frente a las dos cepas de Escherichia coli, en presencia de células (Figuras 3 y 4). Este resultado coincide con otros estudios que concluyen la importancia de la presencia de células para adherirse y colonizar las células epiteliales del antro del estómago y de este modo competir con patógenos como E. coli o H. pylori, con el fin de mejorar la tasa de erradicación del patógeno (Aiba et al., 1998).

Los resultados en general indicaron que la actividad inhibitoria frente a Escherichia coli de las BAL podría deberse a los componentes celulares, exclusión competitiva por nutrientes, a la presencia de ácido láctico y otras sustancias activas a pH bajo. Es decir, que solo la presencia de células y los ácidos orgánicos de algunas de las BAL estudiadas tienen efecto sobre las cepas de éstos patógenos ensayados, $\mathrm{y}$ que algunas pocas de las BAL evaluadas presentaron producción de bacteriocinas.

\section{Conclusiones}

Las BAL aisladas de productos lácteos comerciales $\mathrm{y}$ artesanales presentaron efecto inhibitorio frente a E. coli; mostrando quea nivel artesanal en el sur de Colombia se encuentran productos con potencial probiótico, los cuales requieren ser validados por investigadores adscritos a grupos o centros de investigación con trayectoria y reconocimiento.

\section{Referencias Bibliográficas}

Aiba, Y.; Nobuyuki, S.; Kabir, A.M.; TakagI, A.; Koga, Y. 1998. Lactic acid-mediated suppression of helicobacter pylori by the oral administration of lactobacillus salivarus as a probiotic in a gnotobiotic murine model. The American Journal of gastroenterology, págs.2097-2101.

Amorocho, C. M. 2011. Caracterización y potencial probiótico de bacterias lácticas aisladas de leche de oveja Guirra. Valencia: Universidad Politecnica de Valencia.

AOAC (Association of Official Analytical Chemists), 1997. Methods of Analysis of the AOAC International. 3rd ed. Volumen II. Maryland USA.

Castillo, J. 2001. Elaboración de queso mozzarella con diferentes porcentajes de grasa en la leche de vaca. Trabajo de grado de Ingeniería 75 agrónoma. Guácimo. Costa Rica.: Universidad EARTH. Facultad de Ingeniería agronómica. Pag. 36.

Cortes, E.T.;Peña, N.; Amorocho, C.M. y Gutierrez, N. 2016. Evolucion de parámetros fisicoquímicos de quesillo Huilense, en almacenamiento refrigerado, Biotecnologia en el sector agropecuario y agroindustrial, Vol 14 $\mathrm{N}^{\circ}$ 2, pág 110-118.

Diplock, A.T., Agget, P.J., Ashwell, M., Bornet, F., Fern, E.B. y Roberfroid, M.B. (1999). Scientific concepts of functional foods in Europe. Consensus document. Br J Nutr.

FAO (Food and Agriculture Organization). 2003. Norma del Codex para Leches Fermentadas: CODEX STAN 243-2003. Leche y Productos Lácteos (2da edición). GARCÍA M. E M. \& Fernandez S. I. 2012. Determinación de la humedad de un alimento por un método gravimétrico indirecto por desecación.

http://riunet.upv.es/bitstream/handle/10251/16339/De terminaci\%C3\%B3n20de\%20humedad.

df? sequence $=1$ \&isAllowed=y. $\quad$ (Consultado: Junio de 2016).

Gonzales, E.P. 2010. Caracterización de la composición fisicoquímica del queso fresco 
elaborado artesanalmente en Sehualaca municipio de Minatitlan, Veracruz. Tesis Médico veterinario Zootecnista, Universidad Veracruzana,52 $\mathrm{p}$.

Hudault, S., Liévin, V., Oise Bernet-Camard, M.F., Servin, A.L. 1997. Antagonistic Activity Exerted in vitro and in vivo by Lactobacillus casei (Strain GG) against Salmonella typhimurium C5 Infection. Applied and Environmental Microbiology, págs.513-518.

Isolauri, E., da Costa, H. y Gibson, G. (2000). Working Group on Functional Foods and Probiotics. Boston. pag. 75-81.

Jacobsen, C.N., Nielsen, V.R., Hayford, A.E., Moller, P.L., Michaelsen, K.F., Paerregaard, A., Sandström, B., Tvede, M., Jakobsen, M. 1999. Screening of Probiotic Activities of forty seven strains of Lactobacillus spp. By In vitro techniques and evaluation of the colonization ability of five selected strain in humans. Applied and Environmental Microbiology, 65(11), págs.4949-4956.

Jamuna M.; Jeevaratnam, K. 2004. "Isolation and characterization of lactobacilli from some traditional fermented foods and evaluation of the bacteriocins" J Gen Appl Microbiol 50: 79-90.

Maldonado, R. y Llanca L. 2008. Estudio de la calidad del queso de mano comercializado en el municipio de Girardot, estado Aragua. Venezuela. Revista Científica v.18 N.4. Maracaibo.

Metchnikoff, E. (1908). The prolongation of life, $1^{\mathrm{a}}$ ed. Nueva York: Putnam Sons, 19.

Midolo, P.D.; Lambed, J.R.; Hull, R.; Luo, F. y Grayson, M.L. 1995. In vitro inhibition of Helicobacter pylori NCTC 11637 by organic acids and lactic acid bacteria. Journal of Applied Bacteriology, 79, págs.475-479.

Oliszewski, R.; Cisint, J. C. \& Kairúz, M. 2007. Manufacturing characteristics and shelf life of Quesillo, an Argentinean traditional cheese. Food control, 18(6), 736-741.

Olivares, M.; Díaz-Ropero, M.P.; Martín, R.; Rodríguez, J.M. y Xaus, J. 2006. Antimicrobial potential of four Lactobacillus strains isolated from breast milk. Journal of Applied Microbiology, 101(1), págs.72-79.

Pérez, M.A. \& Echeverry, S. 2015. Análisis microbiológico de alimentos preparados en la vía pública en los alrededores de la universidad surcolombiana mediante el estudio de coliformes. Tesis de pregrado en Licenciatura de Ciencias Biológicas, Universidad Surcolombiana, Neiva, Colombia. 190 p.

Ramírez, J. \& Rodríguez, A. 2012. Caracterización del quesillo colombiano por espectrocolorimetría. Vitae, vol. 19, No. 2, pp.178-185.

Ramírez, C. \& Vélez, J.F. 2012. Quesos frescos: propiedades, métodos de determinación y factores que afectan su calidad.

Schimidt, C.A.P.; Pereira, C.; Dos Anjos, G. y Lucas, S.D.M. 2012. Formulação e avaliação sensorial hedônica de iogurte com polpa de Acerola. Revista Eletrônica Científica Inovação e Tecnologia Vol.1 (5):pag 10-14.

Yuan, K.L. 2006. Microbial biotechnology. Principles and applications Second., National University of Singapore. 